\title{
LASER SCANNING THERMAL PROBE: A NOVEL APPROACH TO NON-DESTRUCTIVE EVALUATION
}

\author{
Jacob Kephart, John Chen, and Hong Zhang \\ Mechanical Engineering Department, Rowan University, 201 Mullica Hill Road, \\ Glassboro, NJ 08028
}

\begin{abstract}
Structural defects such as cracks have recently been identifiable through a new nondestructive evaluation (NDE) technique know as sonic IR or thermosonics. However, these defects are depicted through thermal imagining system as localized "hot spots" indicating a general location of a defect without an accurate portrayal of the dimensions or shape of the defect. This paper demonstrates a new technique called Laser Scanning Thermal Probe, LSTP, which utilizes thermography with the use of heat application in strategic locations to observe spatial heat flow patterns. The LSTP records heat propagation across a defect area previously identified through the thermosonic technique. Thermal gradients will occur as heat traverses the crack and provide information to characterize the crack such as its length and shape.
\end{abstract}

\section{INTRODUCTION}

Recent developments in thermal imaging have proven to be beneficial for use in NDE applications. Techniques using IR cameras are typically classified as two types of systems, active or passive. Passive based thermography uses no additional heating source when observing the object of interest. It simply views any heat emitted from the object without any additional interference. Active thermography has been of particular importance for NDE and various methods using an external heat source, such as heating lamps, have been developed to detect defects in materials [1,2].

Thermosonics is a new thermal NDE technique that injects a short ultrasonic frequency sound wave (typically $0.5 \mathrm{~s}$ at $20-50 \mathrm{kHz}$ ) to induce vibrations in the specimen being examined. This high-frequency-induced vibration causes frictional heating in locations with defects such as cracks, composite disbonds, delaminations, or other locations where two parts are touching without sufficient bonding $[3,4,5,6]$. One main drawback to this technique is the limitation in defect characterization. The characterization of a defect by thermosonics NDE is limited to a localized "hot spot" typically a few degrees Celsius warmer than the bulk material temperature. Specific detail such as the shape of the defect is typically unattainable using this method. Since material defects need to be in close proximity for frictional heating to occur, the tightest locations of a defect are detected by the thermal imaging system very well while, in comparison, loosely contacting defects do not show up as well for the same given energy input as a tighter defect. In general the thermosonic technique is used to detect the existence of a defect, but not detailed information about the shape of the defect. 
This lack of specificity is compensated by speed of detection and provides the motivation to couple thermosonic NDE with thermography, using laser-based heat application to provide both large area and high precision defect characterization. This coupling of methods will provide fast defect location identification through the use of thermosonics, and then specific crack characterization by detailed probing of only the identified defect regions. The application of thermosonics for defect location has already been proven as viable, therefore this paper will deal predominately with the Laser Scanning Thermal Probe for defect characterization.

\section{SAMPLE PREPARATION}

In order to prove the validity of attaining crack defect characterization through heat propagation, a test sample had to be created that would accurately represent an actual defect. In this paper we present test results for two materials, stainless steel and aluminum. The defect chosen was a partially open crack in AISI 316 stainless steel (SS), which provides a good basis for testing this process on metals due to its relatively low thermal conductivity value of $13.4 \frac{\mathrm{W}}{\mathrm{m} \cdot \mathrm{K}}$ at $300 \mathrm{~K}$. The crack manifests as an increase in thermal resistance due to contact resistance of the crack. Since SS is the chosen test material, this relative resistance increase would be lower than in another metal containing the same crack with higher thermal conductivity. In effect, obtaining good results with this sample would be more challenging than a metal sample of higher thermal conductivity.

The metal sample was formed from a 0.115 inch-thick plate and shaped with a notch (see Fig. 1). This notch creates a location of stress concentration so that the defect would start in this region. The holes adjacent to the notch allowed for custom clamps to hold the specimen in a Material Testing Station (MTS) tensile-testing machine without slipping.

Simulated fatigue created a crack in this sample by cyclically loading it at a stress lower than the yield stress until a crack formed. Since the amount of cyclic loading required to cause a fatigue crack is dominated by the amount of time needed to initiate a crack [7], the process was expedited through two measures. The first was in the notch design of the sample, which created a localized stress concentration to be present when loaded in tension. This would ensure crack initiation from the notch point before any other fatigue crack would develop elsewhere. The second means of accelerating the fatigue crack process was to actually initiate a crack by subjecting the sample to a tensile load sufficient enough to exceed the localized yield stress at the stress concentration notch.

Figure 1 shows the results of a simple linear elastic model of the sample, which exhibit the presence of very high von Mises stress at the notch, which exceeds the ultimate strength of the material when the sample is subjected to a static load of $4000 \mathrm{lbs}$.
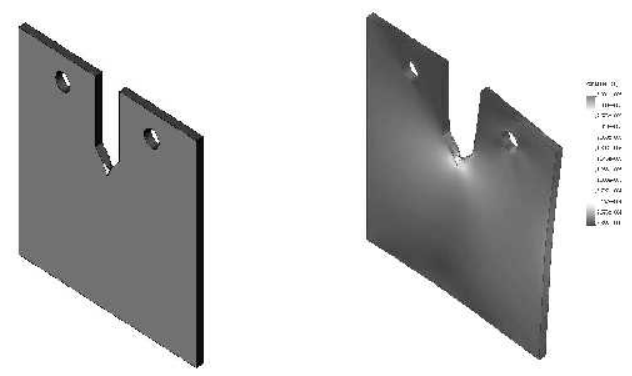

FIGURE 1. (Left) Sample design. (Right) Stress analysis. 


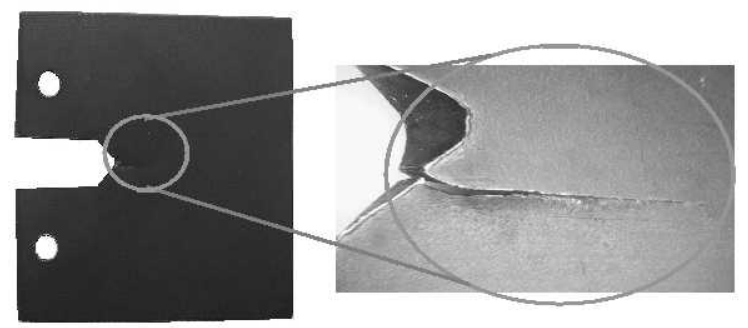

FIGURE 2. (Left) Photograph of cracks sample. (Right) Micrograph of crack.

This means some sort of failure will occur at this site, either deformation or crack initiation. The sample was subjected to such a static load using a MTS machine. This caused a very small tear in the sample, which effectively acted as a fatigue crack initiation site. From this point the sample was cyclically loaded for 40,000 cycles under a tensile force less than the force initially required to initialize the crack. This caused a fatigue crack to propagate 0.360 in. The test sample and a micrograph of the created crack can be seen in Fig. 2.

This crack is very tight toward its termination point and a slightly opened gap can be observed towards the initiation point. This sample was inspected using thermosonics techniques and thermography. The idea of frictional heating due to high frequency vibrations is evident in that where the crack is the tightest a localized hot spot is realized (Fig. 3). This concretely shows, in this sample, that a defect exists and its general location can be obtained through thermosonics. However the only location that can be depicted as a defect is the termination point of the crack, while no information is gained about the remainder of the defect.

Specific characterization of the crack including size and shape would have to be determined using another method. This inspired the idea of applying heat in an approximate and observing the effects the crack has on heat flow.

Another sample was created from 2024-T3 aluminum. The shape of this sample was different than the previously mentioned sample in order to aid in the fatigue cracking. An initial saw cut was made from the middle edge of the sample in order to create stress concentration that will form a closed fatigue crack under cyclic loading. This aluminum sample had a thermal conductivity of $121 \frac{\mathrm{W}}{\mathrm{m} \cdot \mathrm{K}}$ at $300 \mathrm{~K}$ which will cause heat to dissipate more quickly than in the stainless steel sample.

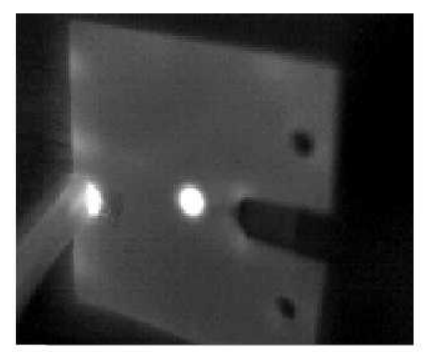

FIGURE 3. Image from the IR camera showing the localized hot spot generated by the thermosonics technique. 


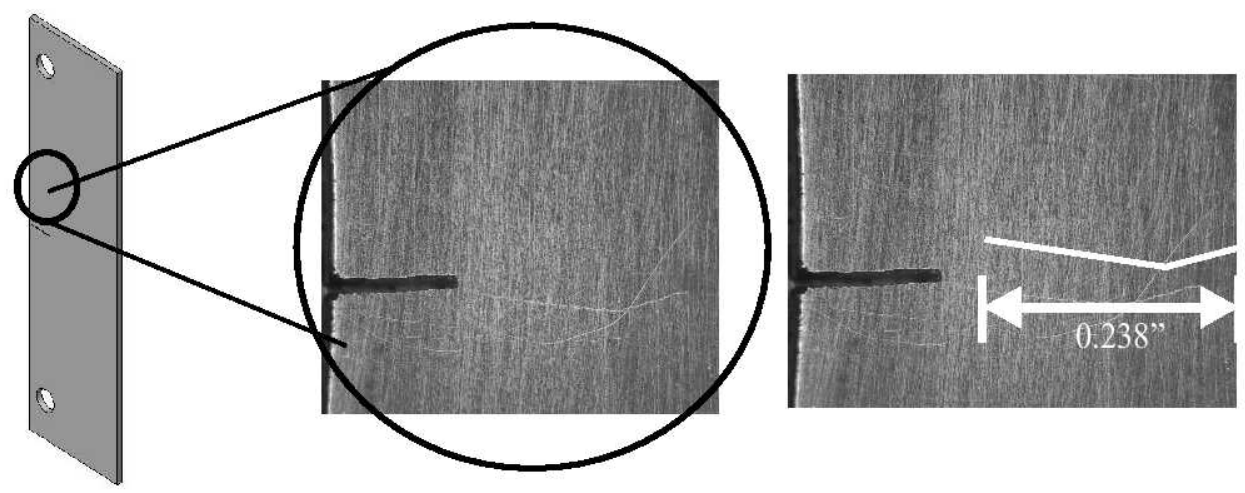

FIGURE 4. (Left) Aluminum sample shape design with small saw cut. (Middle) Micrograph of saw cut and crack (Right) Crack length and shape denoted by white line.

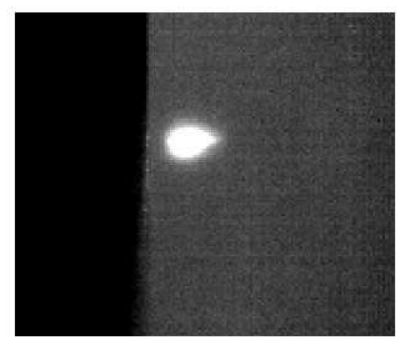

FIGURE 5. Image from IR camera showing localized hot spot for the aluminum sample using thermosonics.

The design of the sample as well as a close-up of the saw cut and crack can be seen in Fig 4. The crack in this sample is closed the entire length of the crack and extends 0.238 in. from the saw cut. This sample was tested with thermosonics and the technique depicts the crack as a localized hot spot, as seen in Fig. 5, without specificity as to the crack shape itself.

\section{THEORETICAL MODEL}

In order to help design the LSTP method, a three-dimensional thermal finite-difference model was developed using actual geometrical parameters of the SS sample. The model uses properties of AISI 316 stainless steel, the sample material used for the simulation.

This model made use of three forms of heat transfer, conduction, radiation, and free convection. Conduction was present in all finite difference elements allowing heat to flow in a three-dimensional space. Natural convection is assumed to take place on the surface nodes of the sample, the same surface that is subjected to the laser heat application. This surface is also treated as a blackbody radiating heat to ambient surroundings. (Radiation effects are small, and thus the neglect of the sample's gray body character causes only very small difference in the results.) The exterior nodes that make up the perimeter of the model sample were treated as insulated since the relative total surface area is small. The surface opposite the heated surface was also treated as insulated since very little convection would occur because of its orientation.

Heat application was modeled as heat addition to a single element of the model at a power of two watts. This heat is added as energy, $E=P^{*} \Delta t$, to the beginning of a time 

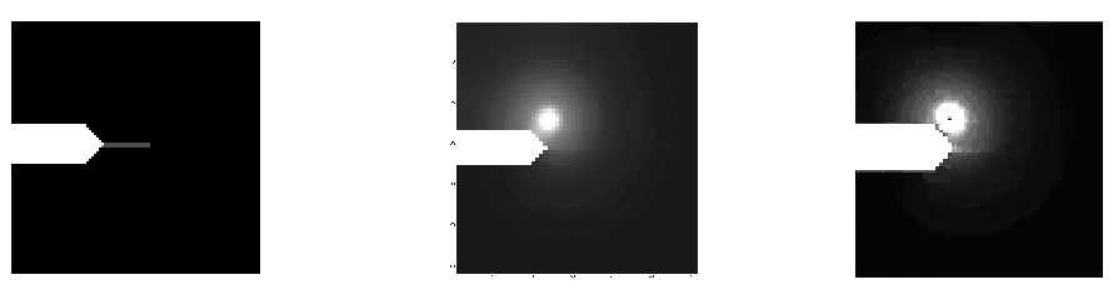

FIGURE 6. (Left) Modeled crack. (Middle) Surface temperature profile. (Right) Gradient processed image.

step allowing some of the total heat added to flow to surrounding nodes by the end of the time step. This eliminates unrealistic spikes in temperature at the nodes with heat addition.

A crack in the material was modeled as a localized decrease in thermal conductivity. It extended from the notch for a distance of 0.5 in. horizontally, as shown in Fig. 6 (left side). The simulated crack itself was not a node or sequence of nodes but a plane of increased thermal resistance for nodes who passed or received heat through it. Figure 6 shows the nodes affected by the crack resistance increase as well as the results (temperature distribution) of a model run with 2 watts of heat input after 60 seconds.

The model visually shows a difference in heat flow in the region of the crack. This is evident by the temperature increase on one side of the crack, and the sharp decrease on the other side. This difference will be observed in greater contrast and the crack depicted through image processing by forming an image using the spatial gradient of the temperature distribution, which is the far-right image in Fig. 6. This model was not adapted to represent the aluminum sample since its purpose was simply an initial verification before and further testing was preformed.

\section{EXPERIMENTAL SETUP}

The LSTP setup consists of four main components: The thermal imaging system, laserbased heat source, robotic arm, and host computer. All are integrated and controlled by the host computer, as depicted in Fig. 7.

The current infrared (IR) camera is a FLIR Systems SC300, which is sensitive to radiation in the 7.5 to 13 micron range and has a focal plane array of $160 \times 120$ pixels. This camera provides a sensitivity of $0.1^{\circ} \mathrm{C}$ in surface temperature change. This IR camera provides the means of recording temperature changes over the area being studied. It is positioned at its minimum focal distance (11.5 in.) directly above the sample showing a viewing area of 5 inches x 3.75 inches. The current focal distance and lens provides a minimum spot size resolution of 0.05 in.

The F3 robot arm (Fig. 8) from CRS Robotics provides the laser positioning and scanning. Use of the robotic arm to apply the heat via a laser provides the flexibility of applying heat in any two-dimensional geometric shape, and onto any surface, whether flat or containing curvature. This latter point will be further utilized in the future as a means to apply heat to a sample that is not planar but a three-dimensional object.

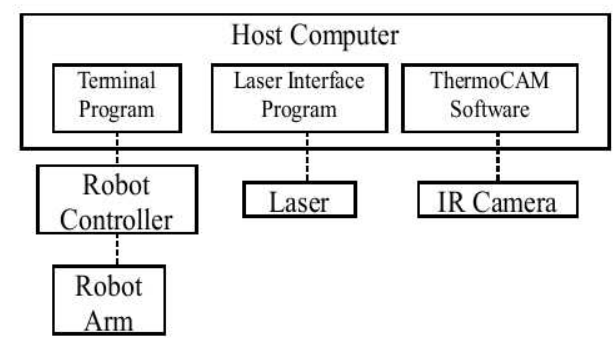

FIGURE 7. Hardware control map of the LSTP system. 


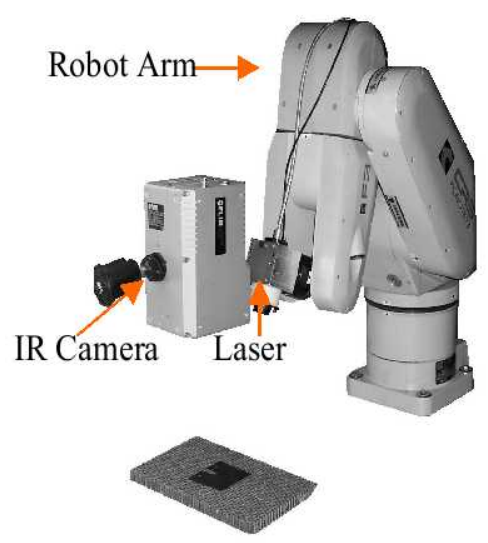

FIGURE 8. IR camera, laser head, and F3 robot arm.

The means of heat application is the laser, which is attached to the robot arm. It is a 9watt solid-state fiber laser that emits light at $1100 \mathrm{~nm}$, which is a shorter wavelength than the IR camera is able to detect. This enables viewing the sample during the heating process without interference or damage to the camera. The heat is applied in a continuous manner for the duration of the scan. The current sample is painted black with enamel paint so effectively all the laser light is absorbed by the sample.

The host computer contains all the software to operate the robot arm, IR camera, and the laser as well as the image processing software and code.

\section{DATA PROCESSING}

The raw images recorded by the camera were in need of image processing in order to obtain images in a format that could be manipulated through pixel count correction, image zeroing, and gradient processing. The image processing flow chart is shown in Fig. 9. The image from the camera recorded all observed heat, including reflected heat from the camera itself. To alleviate the effect of reflected camera heat, the first few frames of a sequence always included the object before any heat was applied. This allowed for the subtraction of the camera's reflected heat from the rest of the image sequences.

In order to enhance the detectablity of a defect, spatial gradient images were employed. For each pixel in a frame of an image sequence, the magnitude of the gradient was calculated relative to both the $\mathrm{x}$ and $\mathrm{y}$ direction, using Eq. (1).

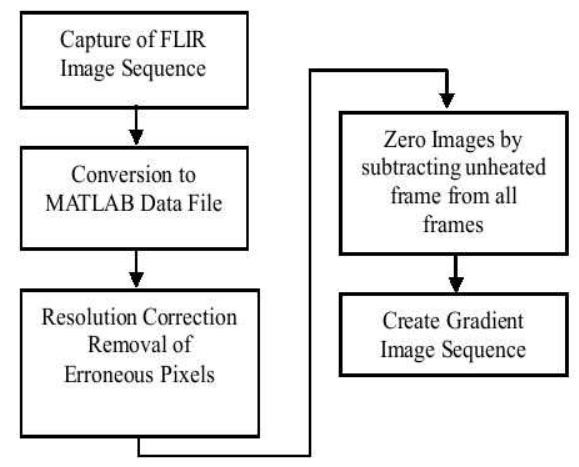

FIGURE 9. Image processing flow chart. 


$$
\Delta P(x, y)=\sqrt{[P(x-1, y)-P(x+1, y)]^{2}+[P(x, y-1)-P(x, y+1)]^{2}},
$$

where $\mathrm{P}(\mathrm{x}, \mathrm{y})$ denotes the temperature at position $(\mathrm{x}, \mathrm{y})$ and $\Delta \mathrm{P}(\mathrm{x}, \mathrm{y})$

denotes the magnitude of the gradient at position $(\mathrm{x}, \mathrm{y})$.

The gradient images were assembled into a three dimensional matrix for easy manipulation, then further displayed as a pseudo-colored image sequence and finally converted into an AVI movie. This allowed for the visual realization of spatial changes in temperature. Visualizing a gradient in heat flow allows for simple detection of either a high intensity heat source (such as our laser), an edge of a object, or a change in thermal conductivity representing a defect.

\section{RESULTS}

The effects of the crack on the heat flow are marginally noticeable in the raw temperature-distribution images but are significantly evident in the gradient processed data. A sequence showing the effects of the heating process after 10,30, and 60 seconds at 2 watts are shown in Fig. 10 for the stainless steel sample. The raw image does not show a significant indication of crack location, shape, or size until 30 seconds after heat initiation. However at this same time, the gradient processed data shows the defect well. The gradient image at 60 seconds shows the defect with greater intensity and contrast.

The location of heat applied on the sample is at a distance of two crack-lengths vertically above the crack and two crack-lengths horizontally shifted to the right from the crack tip. This location provided enough distance away from the heat source so that the gradients due to the heating do not interfere with the gradients across the crack.

A similar test was preformed on the aluminum sample. The power set point of the laser was raised to 9 watts due to aluminum's ability to quickly dissipate heat. The time duration to see the defect is much quicker than that of stainless steel, again due to the difference in thermal conductivity. As seen in Fig. 11 the defect appears much quicker than the SS sample, required only 5 seconds to achieve a good contrast.

Other locations of heat application were tested beyond what was depicted here. In general, the closer the point source of heat is to the defect location, the more discernable the crack when viewed from the raw image. However, when the image is gradient processed, the gradients across the sample are difficult to distinguish from that of the heat source. The shape of the crack is better defined when the heating location is sufficiently far such that the gradients from the heat source have significantly diminished.
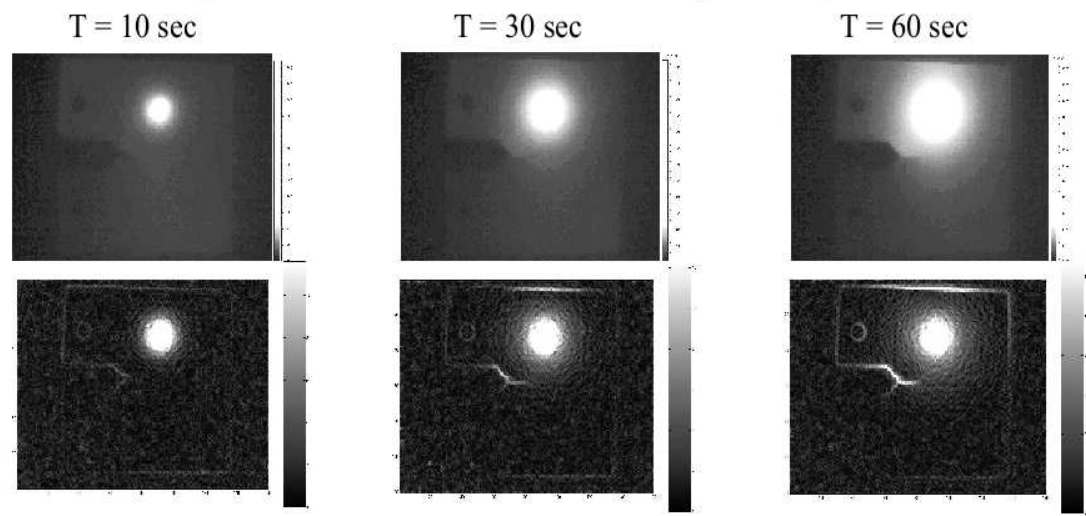

FIGURE 10. (Top) Temperature maps from the IR camera for SS sample. (Bottom) Gradient processed images. 

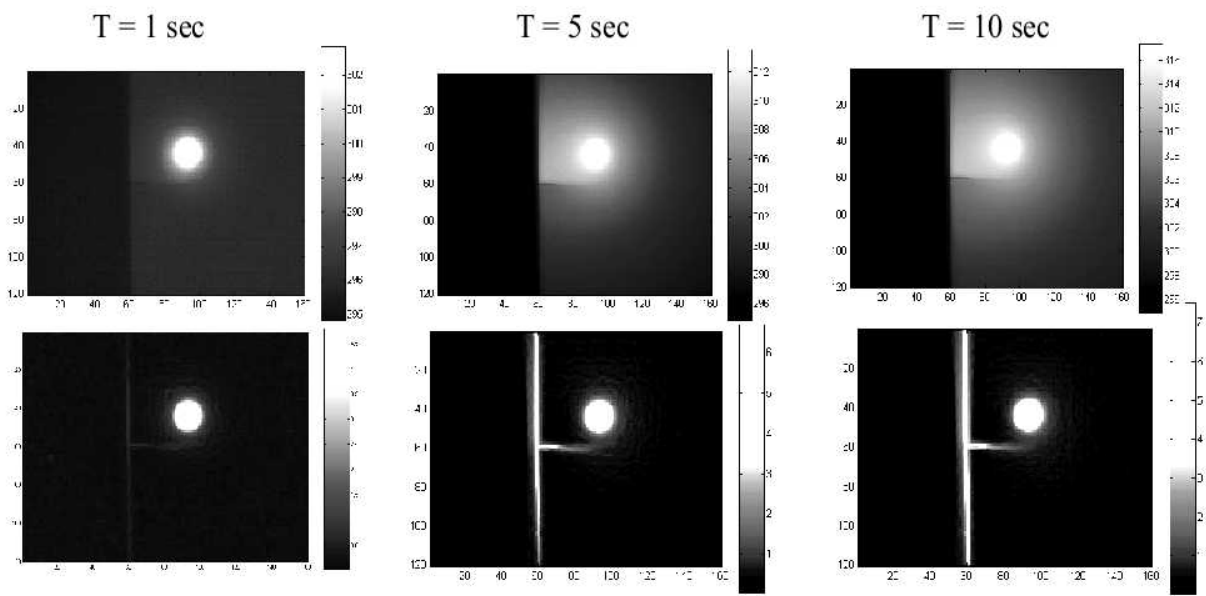

FIGURE 11. (Top) Temperature maps from the IR camera for Al sample. (Bottom) Gradient processed images.

Heating locations to the left of the crack were also tested. These tests showed crack locations very well, but this was expected and is an artifact of the sample design since the laser energy would have no means of heating the opposite side of the crack without first traversing the crack due to the presence of the notch in the sample. This differs from heat locations to the right of the crack in that some heat is able to reach the opposite side of the crack without flowing through it.

\section{CONCLUSION}

The method of point heat application and observing gradient effects has proven itself as a means to characterize crack length in sample testing. The power setting as well as the amount of time needed to discern the crack is a property if the material being inspected. Thermosonics has been proven in the past to identify locations of defects without detailed characterization of the defect itself. Coupling these two methods in an autonomous system will provide the ability to quickly locate defects in a large area using thermosonics and characterize their shape with the laser-scanning probe.

\section{REFERENCES}

1. Maldague, X. "Introduction to NDT by Active Infrared Thermography," Materials Evaluation, 60[9]: $1060-1073,(2002)$.

2. Maldague, X. Nondestructive Evaluation of Materials by Infrared Thermography, SpringerVerlag, New York, (1992).

3. Favro, L.D. Xiaoyan Han, Zhong Ouyang, Gang Sun, Hua Sui, and R.L. Thomas "Infrared imaging of defects heated by a sonic pulse", Review of Scientific Instruments, V. 71, pp. 24182421 , June 2000.

4. Favro, L.D. Xiaoyan Han, Zhong Ouyang, Li Li, Sheng Wang, and R.L. Thomas, "Thermosonic imaging of cracks and delaminations", Progress in Natural Science, V. 11, p133-136, May, 2001.

5. Simon, Daniel L., "Industrial Uses of a New Hybrid Sonic/Infrared NDT Technique", Review of Progress in QNDE-2001, edited by D. O. Thompson et al., AIP Conference Proceedings 615, V. 21a, Melville, New York, 2002, pp. 536-543.

6. Han, Xiaoyan, L.D Favro, Z. Ouyang, and R.L Thomas, "Recent Developments in Thermosonic Crack Detection", Review of Progress in QNDE-2001, edited by D. O. Thompson et al., AIP Conference Proceedings 615, V. 21a, Melville, New York, 2002, pp. 552-557.

7. Yasuhiro Kawaguchi, Yasuharu Shirai, "Fatigue Evaluation of Type 316 Stainless Steel Using Positron Annihilation Lineshape Analysis and Coincidence Positron Lifetime Measurement", Journal of Nuclear Science and Technology V. 39, Oct 2002. 